\title{
Measurement of the parameters of non- stationary gas flows by diode laser absorption spectroscopy in case of high temperature and high pressure
}

\author{
M.A. Bolshov ${ }^{1, *}$, V.V. Liger ${ }^{1}$, Yu.A. Kuritsyn ${ }^{1}$, V.R. Mironenko ${ }^{1}$, Ya.Ya. Ponurovskii ${ }^{2}$, and \\ O.M. Kolesnikov ${ }^{3}$ \\ ${ }^{1}$ Institute for Spectroscopy RAS, 108840 Troitsk, Moscow, Russia \\ ${ }^{2}$ A.M. Prokhorov General Physics Institute RAS, 119991 Moscow, Russia \\ ${ }^{3}$ N.E. Zhukovsky Central Aerohydrodynamic Institute, 140180 Zhukovsky, Russia
}

\begin{abstract}
Experimental version of diode laser absorption spectrometer (DLAS) for contactless measurements of temperature and water vapor concentration in supersonic gas flows is developed. The spectrometer can be used for the measurements of temperature up to $2500 \mathrm{~K}$ and total pressure up to $3 \mathrm{~atm}$. The technique is based on the registration of the transient absorption spectra of a target molecules and fitting of the experimental spectra by the simulated ones constructed using the spectroscopic databases. The temperature is inferred from the ratio of the intensities of the absorption lines with different low energy levels. In gas media with the above parameters the absorption lines are broadened which demands the use of two diode lasers (DL) working in different spectral ranges. The software for selection of the optimal line combinations was developed. The combination of two strong lines in the spectral ranges $1.39 \mu$ and $1.34 \mu$ was selected as the optimal one. The efficiency of the developed technique was exemplified in the first set of the experiments in conditions of real propulsion in Zhukovsky Central Aerohydrodynamic Institute (TsAGI) for the temperatures within (500-2200) K range and total pressure up to $3 \mathrm{~atm}$.
\end{abstract}

\begin{abstract}
Absorption spectroscopy with diode lasers (DLAS) is widely used for diagnostics of gases [1-6]. It is particulary the most popular technique for contactless probing of different combustion zones in mixing gas flows because any traditional thermocouples being inserted in a flow strongly modifies the structure of the flow.

General approach of DLAS technique is based on the measurement of the integral intensities of two lines of a test molecule with different energies of low levels. Assuming the termodynamic equilibrium the ratio of the line intensities $S(T)$ depends only on gas kinetic temperature. In case of low pressure $(<1$ atm.) and temperature $(<1500 \mathrm{~K})$ the absorption lines are relatively narrow and can be spectrally resolved. One can find a spectral interval in near-ir with different absorption lines of water molecules lieing within a
\end{abstract}

* Corresponding author: mbolshov@mail.ru 
range of fast tuning of a diode laser DL $\left(2-3 \mathrm{~cm}^{-1}\right)$. For the diagnostics of such gas medium a single DL can be used.

The situation becomes more problematic if total gas pressure is above $1 \mathrm{~atm}$. and temperature is above $2000 \mathrm{~K}$. In case of high pressure and temperature the absorption lines are broadened and one cannot find a spectral interval of about $3 \mathrm{~cm}^{-1}$ (interval of a fast tuning of a DFB DL) with isolated lines. For such a case two DLs generating in different spectral ranges should be used. Evidently, the spectral intervals with dominating strong lines should be selected. For the above experimental parameters we accounted for the contribution from the neighboring lines and searched for spectral intervals of $3 \mathrm{~cm}^{-1}$ with water molecule lines with close low levels.

The criteria of line selection and different combinations of the absorption lines for DLAS technique are developed. The software for the selection of the lines from the data bases HITRAN-2012 and HITEMP satisfying the criteria of minimal errors of temperature measurement $\delta T$ for the actual experimental error of integral line intensity measurement $\delta S$ is developed. Different algorithms of data processing were checked in numerical experiment.

Specific units of the developed DLAS spectrometer and different algorithms of data processing of the trancient absorption spectra for final evaluation of temperature are described. DLAS enables to measure the temporal evolution of gas flow temperature and water vapor concentration with time resolution of about $1 \mathrm{~ms}$. This resolution is mainly limited by the upper limit of the DL tuning frequency (10 kHz for DFB type lasers). Two optimal spectral intervals for the measurements of gas temperature at total pressure up to $3 \mathrm{~atm}$. and temperature around $2000 \mathrm{~K}$ were selected. The criteria for line selection and the attainable errors in temperature determination are discussed. The developed algorithm of temperature evaluation was tested in numerical experiments. The spectra in both selected intervals were simulated using spectral data bases for the expected parameters of a hot zone - total pressure up to $3 \mathrm{~atm}$., temperature up to $2000 \mathrm{~K}$ and concentration of water vapor of several percents. Then white noise with different mean powers was added to the simulated spectra and these noisy («experimental») spectra were evaluated by different developed algorithms. It was proved that in case of large $\mathrm{S} / \mathrm{N}$ ratio minimal errors in temperature evaluation can be realized if maximal number of absorption lines within the range of DL tuning with integral intensities above $10 \%$ of the most intense line are included in fitting.

The desined and produced new version of DLAS was tested on propulsion system in Zhukovskii Zentral Aerohydrodynamic Institute. In probed zone of the hot gas flow temperature in the range $600-2000 \mathrm{~K}$ and pressure $0.5-3 \mathrm{~atm}$. were measured.

\section{References}

1. M.G. Allen, Meas. Sci. Technol. 9, 545 (1998)

2. R.K. Hanson, Proc. Combust. Inst. 33, 1 (2011)

3. M.A. Bolshov, Y.A. Kuritsyn, Y.V. Romanovskii, Spectrochim. Acta B 106, 45 (2015)

4. C. Schulz, A. Dreizler, V. Ebert, J. Wolfrum, Combustion Diagnostics. Springer Handbook of Experimental Fluid Mechanics (Springer, Berlin, 2007)

5. Z.P. Wang, F. Li, H.B. Gu, X.L. Yu, X.Y. Zhang, Aerosp. Sci. Technol. 42, 169 (2015)

6. M.A. Bolshov, Y.A. Kuritsyn, V.V. Liger, V.R. Mironenko, S.B. Leonov, D.A. Yarantsev, Appl. Phys. B 100, 397 (2010) 